\title{
Impact of human leukocyte antigen mismatch on lung transplant outcome ${ }^{\dagger}$
}

\author{
Yoshito Yamada ${ }^{\mathrm{a},{ }^{\ddagger}}$, Tim Langner $^{\mathrm{a},{ }^{\neq}}$, Ilhan Inci $^{\mathrm{a}}$, Christian Benden $^{\mathrm{b}}$, Macé Schuurmans ${ }^{\mathrm{b}}$, \\ Walter Weder ${ }^{\mathrm{a}}$ and Wolfgang Jungraithmayr ${ }^{\mathrm{a}, *}$
}

a Department of Thoracic Surgery, University Hospital Zurich, Zurich, Switzerland

b Division of Pulmonology, University Hospital Zurich, Zurich, Switzerland

* Corresponding author. Department of Thoracic Surgery, Brandenburg Medical School, Fehrbellinerstrasse 38, 16816 Neuruppin, Germany. Tel: +49-3391-3947410; e-mail: wolfgang.jungraithmayr@mhb-fontane.de (W. Jungraithmayr).

Received 18 June 2017; received in revised form 27 October 2017; accepted 12 November 2017

\begin{abstract}
OBJECTIVES: Human leucocyte antigen (HLA) mismatch between donor and recipient has a differential impact on the outcome after transplant (Tx) among transplantable solid organs. Although the lung is considered a highly antigenic organ, the impact of HLA matching between the donor and the recipient has been shown to be heterogeneous on lung Tx outcome. To provide further evidence that HLA matching should be considered in the decision process prior to lung $T x$, we evaluated the impact of donor/recipient HLA mismatch on the outcome after lung $T x$ at our institution.
\end{abstract}

METHODS: All patients who underwent lung Tx were analysed in this retrospective single-cohort study between 1994 and 2013 for HLA $(-A,-B$ or $-D R)$ matching between the donor and the recipient and their association with overall survival, the incidence of acute cellular rejection (ACR) and the development of chronic lung allograft dysfunction (CLAD).

RESULTS: In total, 371 (197 men) patients were included. Of these, 117 patients had no HLA match (0/6), 143 had a 1/6 match, 77 had 2/6 matches, 28 had 3/6 matches and 6 had 4/6 matches. One hundred and twenty-two (33\%) patients experienced at least 1 episode of ACR and $172(46 \%)$ patients developed CLAD. Univariate analysis showed a significant correlation between HLA mismatch and the development of CLAD, whereas multivariate analysis revealed that the number of HLA matches (hazard ratio $0.76 ; P=0.002$ ), antibodies to cytomegalovirus in either donors or recipients (hazard ratio 1.52; $P=0.036$ ) and donor age (hazard ratio 1.03; $P<0.001$ ) were independent risk factors for the development of CLAD. On the other hand, HLA matches did not correlate with the incidence of ACR and with the overall survival rate.

CONCLUSIONS: The number of HLA mismatches between donors and recipients after lung Tx did not correlate with ACR or with the overall survival. In contrast, HLA mismatch correlated with the development of CLAD and should therefore be considered a risk factor.

Keywords: Lung • Transplantation • Human leucocyte antigen • Acute cellular rejection • Chronic lung allograft dysfunction • Survival

\section{INTRODUCTION}

The impact of human leucocyte antigen (HLA) mismatch between the donor and the recipient on outcome after a transplant (Tx) varies significantly among transplantable solid organs [1]. Although an optimal HLA match has been considered necessary for a favourable outcome in kidney transplants [2], it does not appear to be of similar importance for other organs. The need for HLA matching seems to play a minor role for liver transplants because HLAs on hepatocytes are expressed to a lesser extent compared with the kidney, thereby rendering this organ less antigenic and

tPresented at the 25th European Conference on General Thoracic Surgery, Innsbruck, Austria, 28-31 May 2017.

期e first two authors contributed equally to this work. resulting in a comparable outcome between HLA-mismatched and HLA-matched organ recipients [3].

The lung is considered a particularly highly antigenic organ, dealing with foreign antigens within the circulation upon Tx as well as displaying a large repertoire of receptors against airborne antigens. Compared to outcomes with other solid transplantable organs, the outcome after lung Tx is inferior [4]. The survival of patients who receive lung transplants is mainly hampered by the high incidence of chronic lung allograft dysfunction (CLAD) [5]. Major risk factors for the development of CLAD are the frequency and severity of acute cellular rejection (ACR) but nonimmunological factors [6] also play a role. The impact of HLA mismatch on Tx outcome, including ACR, CLAD and overall survival of patients, has been reported with conflicting results for patients who receive lung transplants [7-9]. Although several 
centres have reported that an HLA mismatch impairs the outcome after a lung $T x$, other groups did not find this correlation [10]. Yet, ACR episodes were found to be associated with HLA mismatch, and the development of CLAD was reported to be increased when more than 2 HLA loci were mismatched upon Tx [11]. Also, the presence of alloantibodies directed against donor HLAs in lung Tx seems to be of increased importance [12-15].

To further strengthen the evidence of HLA mismatch and lung Tx outcome, we provide an analysis of a single-centre cohort of HLA match in patients who underwent lung Tx between 1994 and 2013.

\section{PATIENTS AND METHODS}

\section{Study design}

Clinical parameters and HLA characteristics from donors and recipients who underwent lung Tx in the University Hospital Zurich between 1994 and 2013 were systematically reviewed. Additionally, overall survival, the incidence of ACR and the development of CLAD in recipients who received transplants and the correlation of these parameters with clinical variables including HLA mismatches were analysed. This study was approved by the local ethics committee under the number KEK 2016-02155.

\section{Patients}

A total of 371 patients were included. Only patients with primary transplants (no retransplants) and those with complete HLA data available were included. Clinical variables including HLA donor and recipient data were retrieved from patient charts and donor reports. Two haplotypes of the 3 main genes, HLA-A, HLA-B and HLA-DR, were recorded. In addition, the cytomegalovirus (CMV) status of the patients was evaluated as well as smoking pack years of donors, type of procedure and cold ischaemic time. All patients underwent lung $\mathrm{Tx}$ at the University Hospital Zurich and received standard post-transplant medication, consisting of cyclosporine, prednisone and mycophenolate mofetil and antiviral therapy with ganciclovir as previously described [16]. Two hundred and eightyone $(76 \%)$ patients who had transplants underwent postoperative surveillance bronchoscopy for potential ACR. After receiving a lung $T x$, most patients underwent surveillance bronchoscopy. However, in some cases, no surveillance bronchoscopy was performed because [1] the patient was transferred to another hospital [2], the performance status of the patient was not good enough to perform surveillance bronchoscopy or [3] the patient refused surveillance bronchoscopy. To further identify patients developing CLAD, postoperative lung function tests were also analysed. CLAD was diagnosed if the forced expiratory volume in $1 \mathrm{~s}$ and/or the forced vital capacity declined further to $\leq 80 \%$ of the postoperative best values for at least 3 weeks but was ruled out if specific causes such as ACR, infection or other pathological conditions were present [17]. Patients who died within 3 months postoperatively were not included in the evaluation for CLAD/non-CLAD.

\section{End-points}

The primary end-point of this study was overall survival of the patients who underwent a Tx. Overall survival was recorded in months post-transplant. We selected 1 January 2016 as the date of the event. Secondary end-points were the incidence of ACR and development of CLAD. To reach a conclusion about those end-points, HLA data from the donors and their matching recipients were analysed and cross-matched. In a second step, HLA mismatches were sorted and compared to the time of survival, to the incidence of ACR and to the development of CLAD.

\section{Statistical analysis}

For the evaluation of ACR- and CLAD-free survival, death without $A C R$ and CLAD was not censored. The Kaplan-Meier curves were generated for overall survival and ACR- and CLAD-free survival in patients following lung Tx. A log-rank test was performed to test the differences among the variables. Univariate and multivariate analyses by Cox regression were used for the analysis of risk factors for overall survival and ACR- and CLAD-free survival, because the dependent variable contained not only the event (yes/ no) but also the time frame [18]. Clinical variables used for the multivariate model were those considered significant based on the univariate analysis. In multivariate analysis, quantitative data were regarded as continuous variables. A stepwise forward procedure was used for the selection of the independent variables. A hazard ratio (HR) for significant variables was reported with the $95 \%$ confidence interval $(95 \% \mathrm{Cl})$. A $P$-value of 0.05 or less was considered significant. The statistical analyses were performed with IBM SPSS Statistics, version 22 (IBM Corp, Armonk, NY, USA).

\section{RESULTS}

\section{Human leucocyte antigen mismatches between transplant donor and recipient}

Patient characteristics and perioperative data are outlined in Table 1. Three hundred and forty-seven recipients (94\%) received bilateral lung transplants; 7\%, a single-lung Tx. HLA matches of 6 different haplotypes between donor and recipient of the lung were assigned to 5 different groups: The first group with 117 (32\%) patients comprised patients who had no match in HLA haplotypes between the donor and the recipient (HLA match 0/6). The second group with 143 (39\%) patients comprised patients who had 1 HLA match (HLA match 1/6). The third group, which had 2 HLA matches (HLA match 2/6) comprised 77 (21\%) patients, the fourth group with 3 HLA matches (HLA match 3/6) included $28(8 \%)$ patients and the fifth group with 4 HLA matches (HLA match 4/6) included 6 patients, respectively.

\section{Human leucocyte antigen mismatch is not associated with overall survival}

The 5-year overall survival was $60 \%$, as shown in Fig. 1. The Kaplan-Meier curves in Fig. 2 depict the overall survival stratified by HLA matches. Patients with 4 HLA matches had a better survival rate, although the log-rank test did not show any significant difference. Univariate analysis identified donor age $(P=0.021)$, smoking pack years of donor $(P=0.014)$, recipient age $(P<0.001)$, $C M V$ antibody positivity either in the donor or in the recipient $(P=0.022)$, patients who did not have cystic fibrosis $(P=0.001)$ and unilateral Tx $(P<0.001)$ as unfavourable factors for overall 

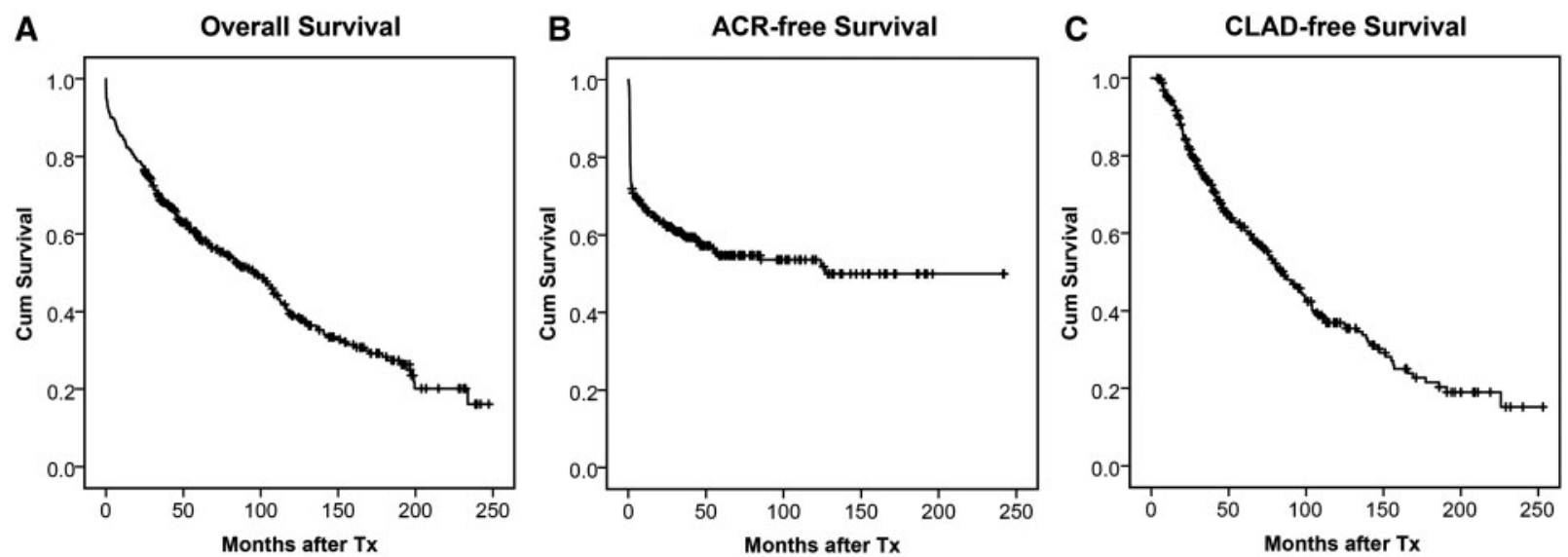

Figure 1: The Kaplan-Meier curves of (A) overall survival, (B) ACR-free survival and (C) CLAD-free survival in all patients. ACR: acute cellular rejection; CLAD: chronic lung allograft dysfunction; Cum: cumulative; Tx: transplant.

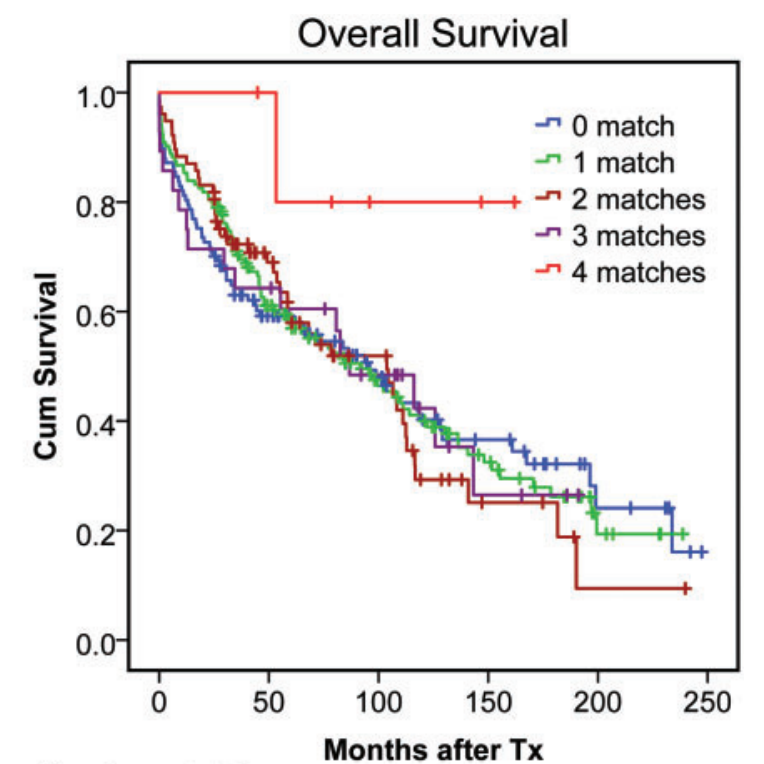

\begin{tabular}{lrrrrrr}
\multicolumn{7}{c}{ Numbers at risk } \\
0 match & 117 & 58 & 35 & 18 & 6 & 0 \\
1 match & 143 & 77 & 44 & 24 & 5 & 0 \\
2 matches & 77 & 39 & 22 & 5 & 1 & 0 \\
3 matches & 28 & 18 & 11 & 3 & 0 & 0 \\
4 matches & 6 & 5 & 2 & 1 & 0 & 0
\end{tabular}

Figure 2: Overall survival of all lung transplant patients with $0,1,2,3$ or 4 human leucocyte antigen matches. Tx: transplant.

95\% $\mathrm{Cl} 1.03-2.25 ; P=0.036)$ were also independent risk factors for the development of CLAD (Table 4).

\section{DISCUSSION}

There is a clear understanding of the central functional role of HLA in the immune response in recipients of solid organ transplants. This fact applies particularly to kidney $T x$ recipients in whom an HLA-A, -B or -DR match between the donor and the recipient is historically compulsory [19]. Less evidence exists for recipients of lung transplants concerning the relation between the level of HLA mismatch and outcome. Donor selection for a lung $T x$ is classically based on clinical urgency, $A B O$ compatibility and donor size. HLA matching is not considered a decisive criterion. However, data from various centres worldwide suggest that the presence and development of anti-HLA antibodies are associated with poorer outcomes after lung Tx. A number of studies emphasize the impact of HLA mismatch on overall outcome. Data from the United Network for Organ Sharing support the finding of the negative association between HLA mismatch and graft and overall survival to be statistically significant in both double and left-lung transplants [20], data that were recently confirmed showing that HLA mismatching is associated with an increased risk of death after lung Tx [21]. These data were also confirmed by others [9]. Our own data suggest that there is an advantage for the overall survival of a patient who has 3 out of 6 matching HLA genes with his or her donor. The differences in survival were not significant between groups; however, a trend indicates the superior survival of patients with at least 4 matching HLA loci. In line with our data, Peltz et al. [12] found that recipients receiving grafts with up to 3 HLA matches had a significantly improved 5-year survival rate compared to patients with a lower number of matching HLA genes. Yet, this study comprised a total of 7127 patients transplanted in the USA between 1994 and 2004 who were included for the survival analysis, which is a much larger patient cohort than our study and may explain the difference in significance. On the other hand, even a large study of 3549 patients showed a modest effect of HLA matching on overall outcome, but the effect of each covariate was small and a definitive conclusion could not be drawn [10].

The main finding of our study on the analysis of all lung $T x$ patients is that the number of HLA mismatches correlates with the development of CLAD, which others have also recognized $[22,23]$. Moreover, when the data were stratified particularly for differences at the HLA-A locus between the donor and the recipient, the incidence of CLAD increased [11, 24]. Alternatively, van den Berg et al. [25] showed specifically that the mismatching on the HLA-DR loci is a risk factor for the development of CLAD and graft loss. However, HLA mismatching itself is not a necessary precondition per se for a poorer outcome. It is rather the presence and development of alloantibodies as a consequence of HLA mismatching that decide the fate of the transplanted organ. In line with this hypothesis, a recent study showed that not the HLA mismatch itself but rather the presence of antibodies impacts graft survival [26]. Also, others could directly show that the development of antibodies to HLA antigens after Tx correlated 
Table 2: Univariate analysis for the development of chronic lung allograft dysfunction and overall survival

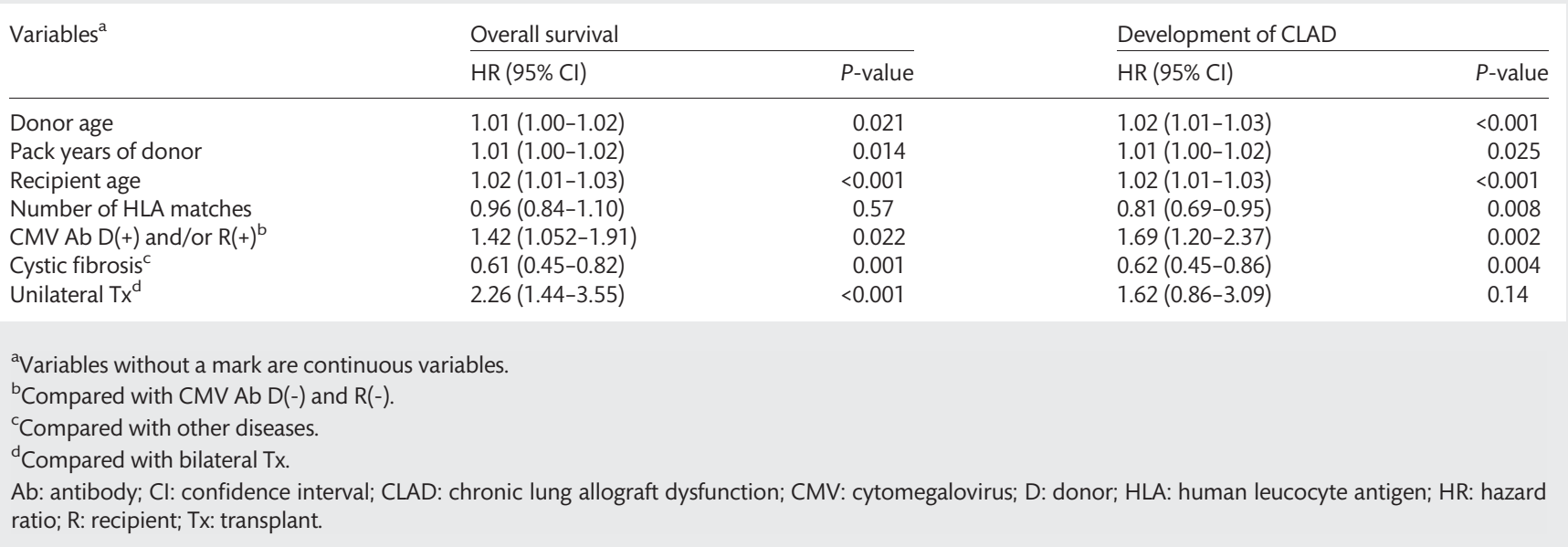

Table 3: Multivariate analysis for overall survival

\begin{tabular}{llr} 
Variables $^{\mathrm{a}}$ & $\mathrm{HR}(95 \% \mathrm{Cl})$ & $P$-value \\
\hline Pack years of donor & $1.01(1.00-1.02)$ & 0.033 \\
Recipient age & $1.02(1.01-1.03)$ & $<0.001$ \\
Unilateral Tx & $1.86(1.08-3.19)$ & 0.025 \\
& \\
& \\
${ }^{\mathrm{a}}$ Variables without a mark are continuous variables. & \\
${ }^{\mathrm{b} C o m p a r e d}$ with bilateral Tx. & \\
Cl: confidence interval; HR: hazard ratio; Tx: transplant.
\end{tabular}

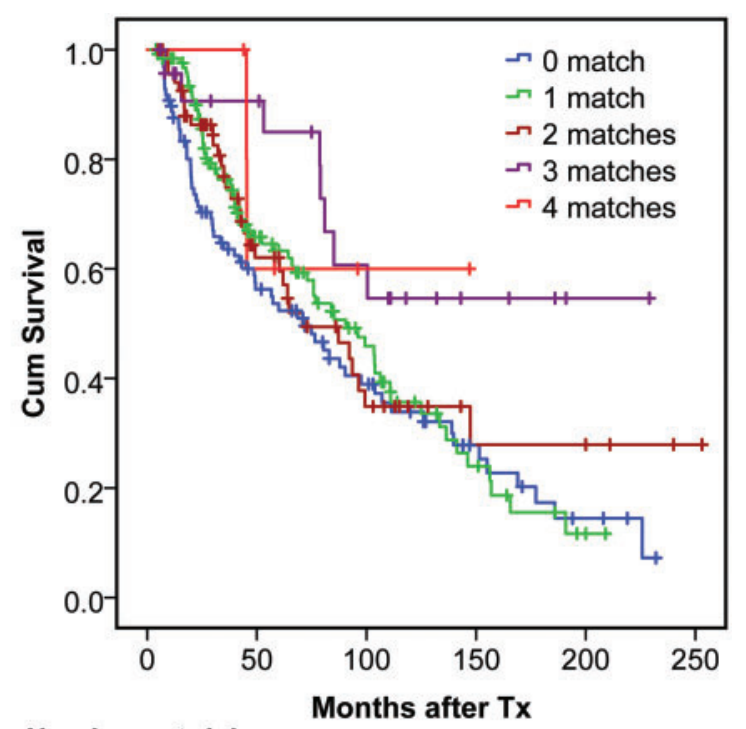

\section{Numbers at risk}

$\begin{array}{lrrrrrl}0 \text { match } & 98 & 45 & 25 & 11 & 4 & 0 \\ 1 \text { match } & 127 & 55 & 28 & 10 & 2 & 0 \\ 2 \text { matches } & 70 & 27 & 12 & 4 & 3 & 1 \\ 3 \text { matches } & 24 & 17 & 10 & 4 & 1 & 0 \\ 4 \text { matches } & 6 & 3 & 1 & 0 & 0 & 0\end{array}$

Figure 3: CLAD-free survival of lung transplant patients with $0,1,2,3$ or 4 human leucocyte antigen matches. CLAD: chronic lung allograft dysfunction; TX: transplant.
Table 4: Multivariate analysis for the development of chronic lung allograft dysfunction

\begin{tabular}{|c|c|c|}
\hline Variables $^{\mathrm{a}}$ & $\mathrm{HR}(95 \% \mathrm{Cl})$ & $P$-value \\
\hline Donor ag & $1.03(1.01-1.04)$ & $<0.001$ \\
\hline Number of HLA matches & $0.76(0.64-0.91)$ & 0.002 \\
\hline $\mathrm{CMV} \mathrm{Ab} \mathrm{D(+)} \mathrm{and/or} \mathrm{R}(+)^{\mathrm{b}}$ & $1.52(1.03-2.25)$ & 0.036 \\
\hline
\end{tabular}

${ }^{\mathrm{a}}$ Variables without a mark are continuous variables.

${ }^{\mathrm{b}}$ Compared with CMV Ab D(-) and R(-).

$\mathrm{Ab}$ : antibody; Cl: confidence interval; CMV: cytomegalovirus; D: donor; HLA: human leucocyte antigen; HR: hazard ratio; R: recipient.

significantly with the development of bronchiolitis obliterans syndrome, the most common form of CLAD. Interestingly, however, the development of antibodies to HLAs did not affect the overall survival rate [27].

Less evidence exists for the correlation of ACR and HLA mismatch. Schulman et al. [8] found that mismatches between donors and recipients at the HLA-DR and HLA-B loci are important risk factors for early high-grade rejections after lung Tx. A study from 2004 [28] showed that HLA-specific antibodies are associated with high-grade lung allograft acute rejection. Wisser et al. [29] found a strong influence of HLA matching on the longterm outcome after lung Tx but no significant correlation of HLA mismatch with ACR or CLAD. In our patients, the existence of 6 out of 6 HLA mismatches did not show a significantly higher incidence of ACR compared to those patients with at least 1 HLA match. Also, there was no advantage in terms of ACR observable for patients with more than $1 \mathrm{HLA}$ match. These dichotomous results underscore the discrepancy between the mismatch of HLA and the presence and effect of antibodies inducing ACR.

\section{Limitations}

One major aspect limits the significance of this study: The limited number of patients with lung transplants does not allow the generation of significant findings for all variables, particularly for 
overall survival, as indicated in the survival curves of HLAmatched patients. Furthermore, the retrospective character of the analysis of data could have potentially excluded confounding variables with a potential risk for data entry errors and missing data points that could have restricted statistical analysis. Furthermore, we were not able to provide the levels of antibodies against HLA for these patients.

\section{CONCLUSION}

HLA-matching should be considered on a regular basis in future studies in order to be able to better characterize and identify the group of patients at increased risk for Tx rejection. Nevertheless, we believe that, with this report, we contribute to the current evidence of the importance of HLA matching by showing that HLA mismatches impact the development of CLAD.

\section{ACKNOWLEDGEMENTS}

The authors thank Professor Burkhardt Seifert, Division of Biostatistics, University of Zurich, for the valuable advice on statistical analysis.

\section{Conflict of interest: none declared.}

\section{REFERENCES}

[1] Mehra NK, Baranwal AK. Clinical and immunological relevance of antibodies in solid organ transplantation. Int J Immunogenet 2016;43: 351-68.

[2] Williams RC, Opelz G, McGarvey CJ, Weil EJ, Chakkera HA. The risk of transplant failure with HLA mismatch in first adult kidney allografts from deceased donors. Transplantation 2016;100:1094-102.

[3] Navarro V, Herrine S, Katopes C, Colombe B, Spain CV. The effect of HLA class I (A and B) and class II (DR) compatibility on liver transplantation outcomes: an analysis of the OPTN database. Liver Transpl 2006; 12:652-8.

[4] Christie JD, Edwards LB, Kucheryavaya AY, Benden C, Dipchand Al, Dobbels $\mathrm{F}$ et al. The Registry of the International Society for Heart and Lung Transplantation: 29th adult lung and heart-lung transplant report2012. J Heart Lung Transplant 2012;31:1073-86.

[5] Verleden GM, Vos R, Vanaudenaerde B, Dupont L, Yserbyt J, Van Raemdonck $D$ et al. Current views on chronic rejection after lung transplantation. Transpl Int 2015;28:1131-9.

[6] Vos R, Verleden SE, Verleden GM. Chronic lung allograft dysfunction: evolving practice. Curr Opin Organ Transplant 2015;20:483-91.

[7] Mangi AA, Mason DP, Nowicki ER, Batizy LH, Murthy SC, Pidwell DJ et al. Predictors of acute rejection after lung transplantation. Ann Thorac Surg 2011;91:1754-62.

[8] Schulman LL, Weinberg AD, McGregor C, Galantowicz ME, Suciu-Foca NM, Itescu S. Mismatches at the HLA-DR and HLA-B loci are risk factors for acute rejection after lung transplantation. Am J Respir Crit Care Med 1998;157:1833-7.

[9] Opelz G, Süsal C, Ruhenstroth A, Döhler B. Impact of HLA compatibility on lung transplant survival and evidence for an HLA restriction phenomenon: a collaborative transplant study report. Transplantation 2010;90: 912-7.

[10] Quantz MA, Bennett LE, Meyer DM, Novick RJ. Does human leukocyte antigen matching influence the outcome of lung transplantation? An analysis of 3, 549 lung transplantations. J Heart Lung Transplant 2000;19: 473-9.

[11] Sundaresan S, Mohanakumar T, Smith MA, Trulock EP, Lynch J, Phelan D et al. HLA-A locus mismatches and development of antibodies to HLA after lung transplantation correlate with the development of bronchiolitis obliterans syndrome. Transplantation 1998;65:648-53.

[12] Peltz M, Edwards LB, Jessen ME, Torres F, Meyer DM. HLA mismatches influence lung transplant recipient survival, bronchiolitis obliterans and rejection: implications for donor lung allocation. J Heart Lung Transplant 2011;30:426-34.

[13] Tait BD, Süsal C, Gebel HM, Nickerson PW, Zachary AA, Claas FH et al. Consensus guidelines on the testing and clinical management issues associated with HLA and non-HLA antibodies in transplantation. Transplantation 2013;95:19-47.

[14] Angaswamy N, Saini D, Ramachandran S, Nath DS, Phelan D, Hachem R et al. Development of antibodies to human leukocyte antigen precedes development of antibodies to major histocompatibility class I-related chain $\mathrm{A}$ and are significantly associated with development of chronic rejection after human lung transplantation. Hum Immunol 2010;71:560-5.

[15] Morrell MR, Pilewski JM, Gries CJ, Pipeling MR, Crespo MM, Ensor CR et al. De novo donor-specific HLA antibodies are associated with early and high-grade bronchiolitis obliterans syndrome and death after lung transplantation. J Heart Lung Transplant 2014;33:1288-94.

[16] Schuurmans MM, Benden C, Inci I. Practical approach to early postoperative management of lung transplant recipients. Swiss Med Wkly 2013; 143:w13773.

[17] Verleden GM, Raghu G, Meyer KC, Glanville AR, Corris P. A new classification system for chronic lung allograft dysfunction. J Heart Lung Transplant 2014;33:127-33.

[18] Bando K, Paradis IL, Komatsu K, Konishi H, Matsushima M, Keena RJ et al. Analysis of time-dependent risks for infection, rejection, and death after pulmonary transplantation. J Thorac Cardiovasc Surg 1995;109: 49-57; discussion 57-59.

[19] Dyer PA, Claas FH. A future for HLA matching in clinical transplantation. Eur J Immunogenet 1997;24:17-28.

[20] Cai J. Double- and single-lung transplantation: an analysis of twenty years of OPTN/UNOS registry data. Clin Transpl 2007;1-8.

[21] Hayes D, Whitson BA, Ghadiali SN, Tobias JD, Mansour HM, Black SM. Influence of HLA mismatching on survival in lung transplantation. Lung 2015;193:789-97.

[22] Harjula AL, Baldwin JC, Glanville AR, Tazelaar H, Oyer PE, Stinson EB et al. Human leukocyte antigen compatibility in heart-lung transplantation. J Heart Transplant 1987;6:162-6.

[23] Chalermskulrat W, Neuringer IP, Schmitz JL, Catellier DJ, Gurka MJ, Randell $\mathrm{SH}$ et al. Human leukocyte antigen mismatches predispose to the severity of bronchiolitis obliterans syndrome after lung transplantation. Chest 2003;123:1825-31.

[24] Brugière $O$, Thabut $G$, Suberbielle $C$, Reynaud-Gaubert $M$, Thomas $P$, Pison $C$ et al. Relative impact of human leukocyte antigen mismatching and graft ischemic time after lung transplantation. J Heart Lung Transplant 2008;27:628-34

[25] van den Berg JW, Hepkema BG, Geertsma A, Koëter GH, Postma DS, de Boer WJ et al. Long-term outcome of lung transplantation is predicted by the number of HLA-DR mismatches. Transplantation 2001;71:368-73.

[26] Sommer W, Hallensleben M, Ius F, Kühn C, Tudorache I, Avsar M et al. Repeated human leukocyte antigen mismatches in lung re-transplantation. Transpl Immunol 2017;40:1-7.

[27] Smith MA, Sundaresan S, Mohanakumar T, Trulock EP, Lynch JP, Phelan $\mathrm{DL}$ et al. Effect of development of antibodies to HLA and cytomegalovirus mismatch on lung transplantation survival and development of bronchiolitis obliterans syndrome. J Thorac Cardiovasc Surg 1998;116:812-20.

[28] Girnita AL, McCurry KR, Iacono AT, Duquesnoy R, Corcoran TE, Awad M et al. HLA-specific antibodies are associated with high-grade and persistent-recurrent lung allograft acute rejection. J Heart Lung Transplant 2004;23:1135-41.

[29] Wisser W, Wekerle T, Zlabinger G, Senbaclavaci O, Zuckermann A, Klepetko $W$ et al. Influence of human leukocyte antigen matching on long-term outcome after lung transplantation. J Heart Lung Transplant 1996;15:1209-16. 